\title{
CMR evaluation of change in myocardial strain following transcatheter aortic valve implantation
}

Akhlaque Uddin*, Timothy A Fairbairn, Peter P Swoboda, Ananth Kidambi, Manish Motwani, David P Ripley, Tarique A Musa, Adam K McDiarmid, Sven Plein, John P Greenwood

From 17th Annual SCMR Scientific Sessions

New Orleans, LA, USA. 16-19 January 2014

\section{Background}

Transcatheter Aortic Valve Implantation (TAVI) is the treatment of choice for patients with severe symptomatic aortic stenosis (AS) who are at high surgical risk. AS results in changes in myocardial strain and twist. Myocardial strain, strain rate and twist can be measured with myocardial tagging CMR. It is not known how TAVI affects LV diastolic and systolic function as measured by CMR tagging.

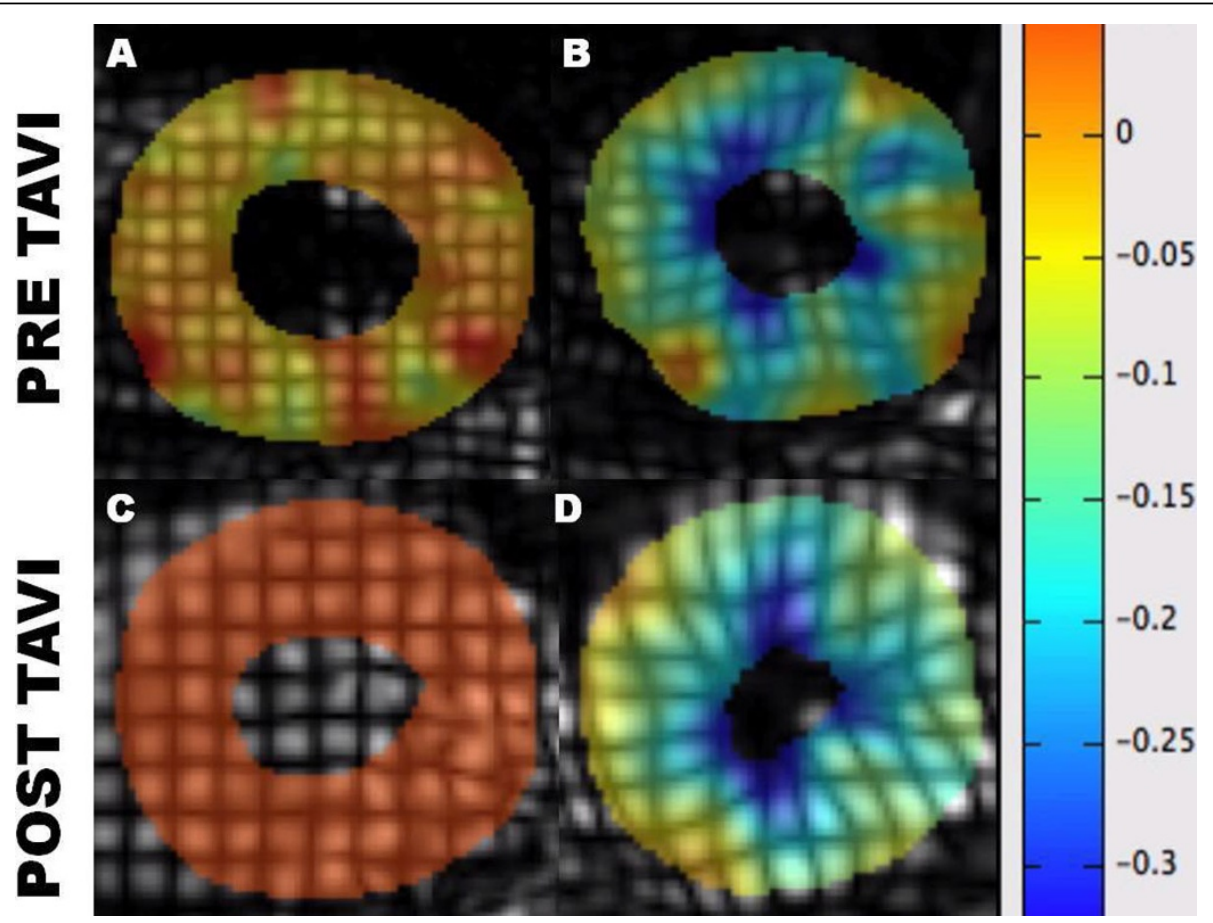

Figure 1 Example of inTag analysis using complementary spatial modulation of magnetization (CSPAMM). Diastole (A, C) and systole (B, D). 


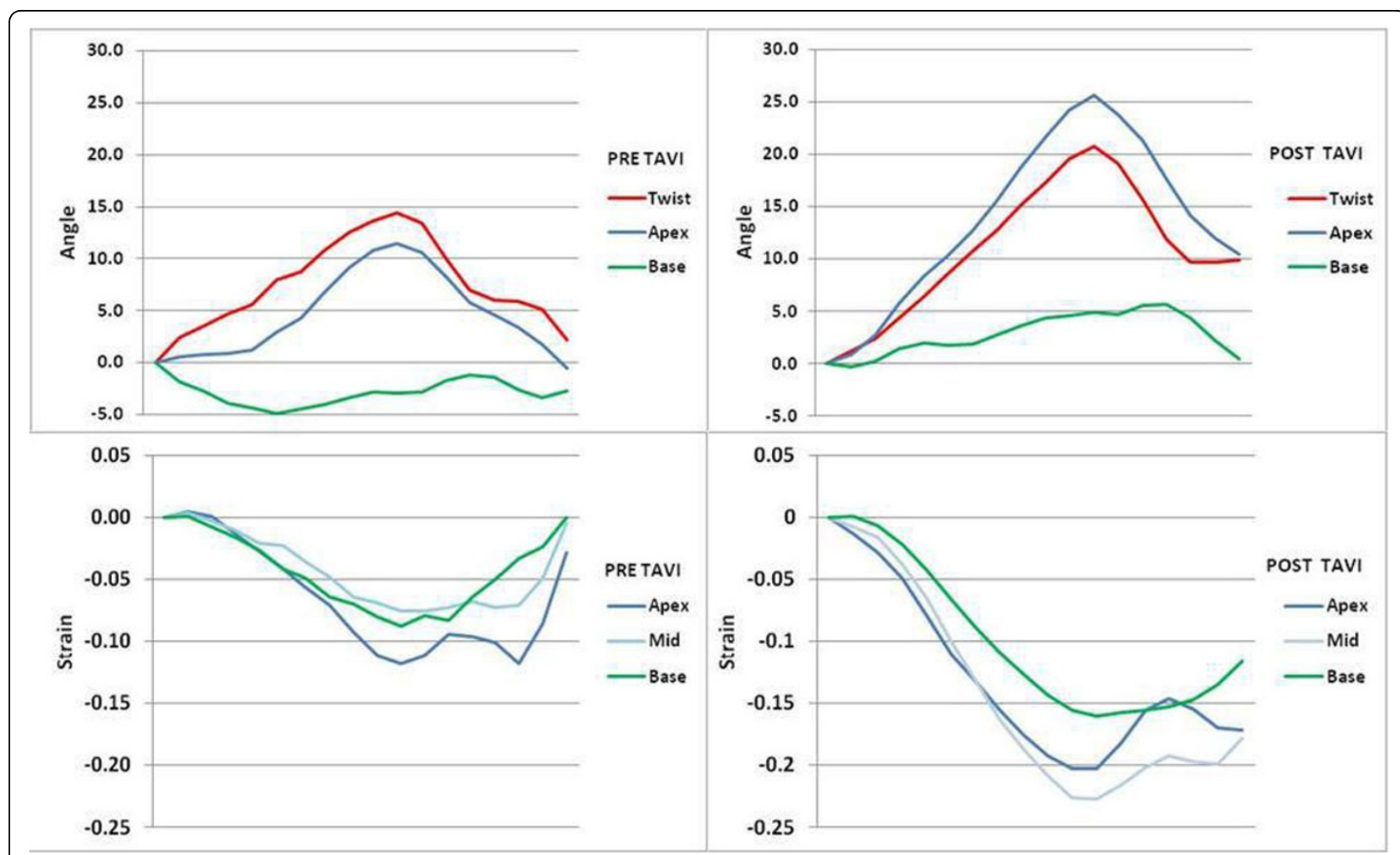

Figure 2 Change in twist and circumferential strain. A) Twist pre-TAVI. B) Twist post TAVI. Circumferential strain pre- (C) and post-TAVI (D).

\section{Objective}

To determine changes in myocardial strain following TAVI.

\section{Methods}

25 patients (age $80 \pm 6$ years, male 14 (56\%), EuroSCORE $22 \pm 14)$ underwent CMR (1.5T, Intera CV, Philips Healthcare) before and 6 months after TAVI. Tagged cine images were acquired at the apex, mid-ventricle and base with a complementary spatial modulation of magnetization (CSPAMM) pulse sequence (FOV $300 \mathrm{~mm}$, matrix $128 \times 128$, slice thickness $10 \mathrm{~mm}$, tag separation $8 \mathrm{~mm}, 18$ phases, typical TR/TE $30 \mathrm{~ms} / 6 \mathrm{~ms}$, flip angle $25^{\circ}$ ). Data were analysed using inTag ${ }^{\odot}$ software (Creatis, Lyon, Fr). Endocardial and epicardial contours were drawn and segmented into 3 layers.

\section{Results}

Following TAVI, peak Lagrangian circumferential strain increased in the mid-LV $(-0.19 \pm 0.06$ vs. $-0.22 \pm 0.07$, $P=0.03)$. There was no significant change in apical $(-0.20 \pm 0.06$ vs. $-0.20 \pm 0.07, \mathrm{P}=0.81)$ or basal $(-0.19 \pm$ 0.06 vs. $-0.20 \pm 0.06, \mathrm{P}=0.24)$ circumferential strain. LV twist decreased after TAVI $\left(17.2 \pm 4.9^{\circ}\right.$ vs. $13.9 \pm$ $\left.5.4^{\circ}, \mathrm{P}=0.04\right)$ and peak systolic strain rate increased $\left(-0.92 \pm 0.24 \mathrm{~S}^{-1}\right.$ vs. $\left.-1.11 \pm 0.23 \mathrm{~S}^{-1}, \mathrm{P}=0.001\right)$ but there was no change in early diastolic strain rate $(0.91 \pm$ $0.51 \mathrm{~S}^{-1}$ vs. $\left.0.88 \pm 0.43 \mathrm{~S}^{-1}, \mathrm{P}=0.98\right)$.

\section{Conclusions}

TAVI results in an improvement in mid-LV circumferential strain, and a decrease in myocardial twist. Systolic strain rate increased following TAVI but there was no significant change in diastolic strain rate. This suggests that whilst systolic function improves, diastolic function does not improve in severe AS at 6 months post-TAVI.

\section{Funding}

SP is funded by a British Heart Foundation fellowship (FS/10/62/28409). SP and JPG receive an educational research grant from Philips Healthcare.

Published: 16 January 2014

doi:10.1186/1532-429X-16-S1-P260

Cite this article as: Uddin et al:: CMR evaluation of change in myocardial strain following transcatheter aortic valve implantation. Journal of Cardiovascular Magnetic Resonance 2014 16(Suppl 1):P260. 